\title{
Solving an Inverse Sturm-Liouville Problem by a Lie-Group Method
}

\author{
Chein-Shan Liu', 2 \\ ${ }^{1}$ Department of Mechanical and Mechatronic Engineering, Taiwan Ocean University, \\ Keelung 20224, Taiwan \\ ${ }^{2}$ Department of Harbor and River Engineering, Taiwan Ocean University, Keelung 20224, Taiwan
}

Correspondence should be addressed to Chein-Shan Liu, csliu@mail.ntou.edu.tw

Received 8 September 2007; Revised 21 December 2007; Accepted 29 January 2008

Recommended by Colin Rogers

Solving an inverse Sturm-Liouville problem requires a mathematical process to determine unknown function in the Sturm-Liouville operator from given data in addition to the boundary values. In this paper, we identify a Sturm-Liouville potential function by using the data of one eigenfunction and its corresponding eigenvalue, and identify a spatial-dependent unknown function of a SturmLiouville differential operator. The method we employ is to transform the inverse Sturm-Liouville problem into a parameter identification problem of a heat conduction equation. Then a Lie-group estimation method is developed to estimate the coefficients in a system of ordinary differential equations discretized from the heat conduction equation. Numerical tests confirm the accuracy and efficiency of present approach. Definite and random disturbances are also considered when comparing the present method with that by using a technique of numerical differentiation.

Copyright (C) 2008 Chein-Shan Liu. This is an open access article distributed under the Creative Commons Attribution License, which permits unrestricted use, distribution, and reproduction in any medium, provided the original work is properly cited.

\section{Introduction}

The problem to describe the interaction between colliding particles is a fundamental one in the physics of particle, where the identification of Schrödinger operator is utmost important. It is one sort of the inverse Sturm-Liouville problems which have various versions. Among them, the best known one is studied by Gel'fand and Levitan [1], in which the potential function is uniquely determined by spectral function. McLaughlin [2] has given an analytical method to treat this type of inverse problems.

There were many works to develop algorithms for solving the inverse Sturm-Liouville problem of reconstructing potential function from eigenvalues [3, 4], which is known as the inverse spectral problem or inverse eigenvalue problem [5]. On the other hand, McLaughlin [6] first noted that it is possible to obtain the potential function and boundary conditions using 
only the set of nodal points. This interesting problem has soon been known as the inverse nodal problem [7-10].

Numerical methods often transform the inverse Sturm-Liouville problem into an inverse eigenvalue problem of a certain matrix [11]. However, many of these discretizations into a matrix form have higher eigenvalues significantly differening from those of true eigenvalues. As a consequence, the inverse algorithms based on these discretizations require careful implementation $[3,4]$.

In this study, the data of spectral function is chosen in order to identify a spatialdependent potential function; hence, the present inverse Sturm-Liouville problem is less difficult than those considered in [3, 4, 6-10].

First, we transform the inverse Sturm-Liouville problem into a parameter identification problem governed by a parabolic type partial differential equation (PDE). Then, a one-step group-preserving scheme (GPS) for a semidiscretization of that PDE is established, which can be used to derive a closed-form solution of the estimated potential function at discretized spatial points. This type approach is first time appeared in the literature.

Let us consider a second-order ordinary differential equation (ODE) describing the Sturm-Liouville boundary value problem:

$$
\begin{aligned}
\frac{d}{d x}\left[p(x) \frac{d y}{d x}\right]+[q(x) & +\lambda r(x)] y=F(x) \quad \text { in } x_{0} \leq x \leq x_{f}, \\
y & =A_{0} \quad \text { at } x=x_{0}, \\
y & =B_{0} \quad \text { at } x=x_{f} .
\end{aligned}
$$

The direct problem for the given conditions in (1.2) and (1.3) and the given functions $p(x)$, $q(x), r(x)$, and $F(x)$ is to find the solution $y(x)$ of the second-order boundary value problem (BVP). Specifically, when $F(x)=0$, we have a Sturm-Liouville problem to determine the eigenvalue $\lambda$ and eigenfunction $y(x)$.

The present inverse problem of Sturm-Liouville is to estimate $q(x)$ by using the information of one eigenfunction $y(x)$ and its corresponding eigenvalue $\lambda$, and for the Sturm-Liouville differential operator is to estimate $p(x)$ by using the data of $y(x)$ when $q(x)=r(x)=0$.

For the case when $p\left(x_{f}\right)$ is known and $q(x)=r(x)=0$ in (1.1), we propose a noniterative method to calculate $p(x)$ at discretized spatial points. This problem could also be solved by the iterative method given by Keung and Zou [12] for the elliptic problem $\nabla \cdot(p \nabla u)=F$. Some of the numerical examples in Keung and Zou [12] involve Sturm-Liouville problems, but the method proposed here requires less computation for these problems.

For the case of $q(x)=r(x)=0$ from (1.1), it follows directly that

$$
p(x) y^{\prime}(x)=p\left(x_{0}\right) y^{\prime}\left(x_{0}\right)+\int_{x_{0}}^{x} F(s) d s
$$

If $y^{\prime}(x), p\left(x_{0}\right)$ and $y^{\prime}\left(x_{0}\right)$ are available, the above equation simply gives the unknown parameter $p(x)$ by dividing both sides by $y^{\prime}(x)$. However, because $y(x)$ is usually not given in a closed-form and is given discretizedly under a perturbation by noise, we require a numerical technique to find $y^{\prime}(x)$. As mentioned by Li [13], several techniques were developed to construct useful difference formulas for numerical derivatives (NDs). In addition to the references in [13], we also mention the book by Shu [14]. Among the many NDs, we only employ the 
method by Ahn et al. [15] to compare it with our new method for numerical examples given in Section 6. Ahn et al. [15] have used a Volterra integral equation of the second kind to derive the following numerical derivative of a function $f(x)$ under noise denoted by $f_{\delta}(x)$ :

$$
f_{\delta}^{\prime}(x)=\frac{-1}{\alpha^{2}} \exp \left(\frac{-x}{\alpha}\right) \int_{0}^{x} \exp \left(\frac{s}{\alpha}\right) f_{\delta}(s) d s+\frac{f_{\delta}(x)}{\alpha}
$$

where $\alpha$ is a regularized parameter and $f_{\delta}^{\prime}(x)$ is a numerical derivative of $f_{\delta}(x)$.

Lie-group is a differentiable manifold, endowed a group structure that is compatible with the underlying topology of manifold. The main purpose of Lie-group solver is for providing a better algorithm that retains the orbit generated from numerical solution on the manifold which associated with the Lie-group [16,17]. The retention of Lie-group structure under discretization is vital in the recovery of qualitatively correct behavior in the minimization of numerical error $[18,19]$.

Liu [20] has extended the GPS developed in [19] for ODEs to solve the BVPs, and the numerical results reveal that the GPS is a rather promising method to effectively solve the twopoint BVPs. In that construction of Lie-group method for the calculations of BVPs, Liu [20] has introduced the idea of one-step GPS by utilizing the closure property of Lie-group, and hence, the new shooting method has been named the Lie-group shooting method.

It should be stressed that the one-step property of Lie-group is usually not shared by other numerical methods because those methods do not belong to the Lie-group type. This important property has been used by Liu [21] to establish a one-step estimation method to estimate the temperature-dependent heat conductivity, and then extended to estimate heat conductivity and heat capacity [22-24]. Its group structure gives the Lie-group method a great advantage over other numerical methods. It is a powerful technique to solve the inverse problem of parameter identification.

This paper is arranged as follows. We introduce a novel approach of an inverse SturmLiouville problem in Section 2 by transforming it into an identification problem of a parabolic type PDE, and then discretizing the PDE into a system of ODEs at discretized spatial points. In Section 3, we give a brief sketch of the GPS for ODEs for a self-content reason. Due to its good property of Lie-group, we will propose a one-step GPS which can be used to identify the parameters appeared in the PDE. The resulting algebraic equation is derived in Section 4 when we apply the one-step GPS to identify $q(x)$. We demonstrate how the Lie-group theory can help us to solve the parameter estimation equation in a closed-form. In Section 5, we turn our attention to the estimation of $p(x)$ which leads again to a closed-form solution of the parameter $p(x)$ at discretized spatial points. In Section 6, several numerical examples are examined to test the Lie-group estimation method (LGEM). Finally, we give conclusions in Section 7.

\section{A novel approach}

\subsection{Transformation into a PDE}

In the solution of linear PDE, a common technique is the separation of variables from which the PDE is transformed into ODEs. We may reverse this process by considering

$$
u(x, t)=(1+t) y(x)
$$


such that (1.1)-(1.3) are changed to

$$
\begin{aligned}
\frac{\partial u(x, t)}{\partial t}=\frac{\partial}{\partial x}\left[p(x) \frac{\partial u(x, t)}{\partial x}\right] & +[q(x)+\lambda r(x)] u(x, t)+h(x, t) \\
\text { in } x_{0} \leq x \leq x_{f}, 0<t \leq T, & \\
u\left(x_{0}, t\right) & =A_{0}(1+t), \\
u\left(x_{f}, t\right) & =B_{0}(1+t), \\
u(x, 0) & =y(x),
\end{aligned}
$$

where $h(x, t)=y(x)-(1+t) F(x)$, and the last initial condition follows from (2.1) directly.

Equation (2.2) is a heat conduction equation, where we are attempting to estimate $p(x)$ or $q(x)$ under a given source $h(x, t)$.

\subsection{Semidiscretization}

The semidiscrete procedure of PDE produces a coupled system of ODEs. For the onedimensional heat conduction (2.2), we adopt the numerical method of line to discretize the spatial coordinate $x$ by

$$
\begin{gathered}
\left.\frac{\partial u(x, t)}{\partial x}\right|_{x=x_{i}=x_{0}+i \Delta x}=\frac{u_{i+1}(t)-u_{i}(t)}{\Delta x} \\
\left.\frac{\partial^{2} u(x, t)}{\partial x^{2}}\right|_{x=x_{i}=x_{0}+i \Delta x}=\frac{u_{i+1}(t)-2 u_{i}(t)+u_{i-1}(t)}{(\Delta x)^{2}}
\end{gathered}
$$

where $\Delta x=\left(x_{f}-x_{0}\right) /(n+1)$ is a uniform discretization spacing length, and $u_{i}(t)=u\left(x_{0}+i \Delta x, t\right)$ for a simple notation. Such that (2.2) can be approximated by

$$
\begin{aligned}
\dot{u}_{i}(t)= & \frac{p_{i}}{(\Delta x)^{2}}\left[u_{i+1}(t)-2 u_{i}(t)+u_{i-1}(t)\right] \\
& +p_{i}^{\prime} \frac{u_{i+1}(t)-u_{i}(t)}{\Delta x}+\left(q_{i}+\lambda r_{i}\right) u_{i}(t)+h_{i}(t), \quad i=1, \ldots, n,
\end{aligned}
$$

where $p_{i}=p\left(x_{i}\right), p_{i}^{\prime}=p^{\prime}\left(x_{i}\right), q_{i}=q\left(x_{i}\right), r_{i}=r\left(x_{i}\right)$, and $h_{i}(t)=y_{i}-(1+t) F_{i}$ with $y_{i}=y\left(x_{i}\right)$ and $F_{i}=F\left(x_{i}\right)$.

When $i=1$, the term $u_{0}(t)$ is determined by boundary condition (2.3) with $u_{0}(t)=A_{0}(1+$ $t$ ). Similarly, when $i=n$, the term $u_{n+1}(t)$ is determined by boundary condition (2.4) with $u_{n+1}(t)=B_{0}(1+t)$. The next step is to advance the solution from a given initial condition to a desired time $T$. However, (2.8) has totally $n$ coupled linear ODEs for the $n$ variables $u_{i}(t), i=$ $1, \ldots, n$, which can be numerically integrated to obtain $u_{i}(T)$.

In this section, we have transformed the inverse Sturm-Liouville problem in (1.1) into an inverse parameter identified problem for the PDE in (2.2), and finally to an estimation of $n$ coefficients $q_{i}$ or $p_{i}$ in the $n$-dimensional linear ODEs system. The data required in the estimation are the discretization of $y(x)$ at discretized spatial points, that is, $y_{i}=y\left(x_{i}\right)$. 


\section{GPS for differential equations system}

\subsection{Group-preserving scheme}

Upon letting $\mathbf{u}=\left(u_{1}, \ldots, u_{n}\right)^{\mathrm{T}}$ and denoting $\mathbf{f}$ the right-hand side of $(2.8)$, we can write it as a vector form:

$$
\dot{\mathbf{u}}=\mathbf{f}(\mathbf{u}, t), \quad \mathbf{u} \in \mathbb{R}^{n}, t \in \mathbb{R} .
$$

Liu [19] has embedded (3.1) into an augmented dynamical system, which is concerned with not only the evolution of state variables but also the evolution of the magnitude of the state variables vector:

$$
\frac{d}{d t}\left[\begin{array}{c}
\mathbf{u} \\
\|\mathbf{u}\|
\end{array}\right]=\left[\begin{array}{cc}
\mathbf{0}_{n \times n} & \frac{\mathbf{f}(\mathbf{u}, t)}{\|\mathbf{u}\|} \\
\frac{\mathbf{f}^{\mathrm{T}}(\mathbf{u}, t)}{\|\mathbf{u}\|} & 0
\end{array}\right]\left[\begin{array}{c}
\mathbf{u} \\
\|\mathbf{u}\|
\end{array}\right]
$$

Equation (3.2) gives us a Minkowskian structure of the augmented state variables of $\mathbf{X}:=\left(\mathbf{u}^{\mathrm{T}},\|\mathbf{u}\|\right)^{\mathrm{T}}$ to satisfy the cone condition:

$$
\mathbf{X}^{\mathrm{T}} \mathbf{g} \mathbf{X}=0
$$

where

$$
\mathbf{g}=\left[\begin{array}{cc}
\mathbf{I}_{n} & \mathbf{0}_{n \times 1} \\
\mathbf{0}_{1 \times n} & -1
\end{array}\right]
$$

is a Minkowski metric, $\mathbf{I}_{n}$ is the identity matrix of order $n$, and the superscript T stands for the transpose. In terms of $(\mathbf{u},\|\mathbf{u}\|)$, (3.3) becomes

$$
\mathbf{X}^{\mathrm{T}} \mathbf{g X}=\mathbf{u} \cdot \mathbf{u}-\|\mathbf{u}\|^{2}=\|\mathbf{u}\|^{2}-\|\mathbf{u}\|^{2}=0,
$$

where the dot between two $n$-dimensional vectors denotes their Euclidean inner product. The cone condition is thus the most natural constraint that we can impose on the dynamical system (3.2).

Consequently, we have an $n+1$-dimensional augmented system:

$$
\dot{\mathbf{X}}=\mathbf{A X}
$$

with a constraint (3.3), where

$$
\mathbf{A}:=\left[\begin{array}{cc}
\mathbf{0}_{n \times n} & \frac{\mathbf{f}(\mathbf{u}, t)}{\|\mathbf{u}\|} \\
\frac{\mathbf{f}^{\mathrm{T}}(\mathbf{u}, t)}{\|\mathbf{u}\|} & 0
\end{array}\right]
$$


satisfying that

$$
\mathbf{A}^{\mathrm{T}} \mathbf{g}+\mathbf{g A}=\mathbf{0}
$$

is a Lie algebra $s o(n, 1)$ of the proper orthochronous Lorentz group $S O_{o}(n, 1)$.

Although the dimension of the new system is raised one more, it has been shown that the new system has an advantage to permit the group-preserving scheme (GPS) given as follows [19]:

$$
\begin{gathered}
\mathbf{X}_{\ell+1}=\mathbf{G}(\ell) \mathbf{X}_{\ell}, \\
\mathbf{G}^{\mathrm{T}} \mathbf{g G}=\mathbf{g}, \\
\operatorname{det} \mathbf{G}=1, \\
G_{0}^{0}>0,
\end{gathered}
$$

where $G_{0}^{0}$ is the 00th component of $\mathbf{G}, \mathbf{X}_{\ell}$ denotes the numerical value of $\mathbf{X}$ at the discrete time $t_{\ell}$, and $\mathrm{G}(\ell) \in S O_{o}(n, 1)$ is the group value of $\mathrm{G}$ at a time $t_{\ell}$. If $\mathrm{G}(\ell)$ satisfies the properties in (3.10)-(3.12), then $\mathbf{X}_{\ell}$ satisfies the cone condition in (3.3).

The Lie-group $\mathbf{G}$ can be generated from $\mathbf{A} \in s o(n, 1)$ by an exponential mapping,

$$
\mathbf{G}(\ell)=\exp [\Delta t \mathbf{A}(\ell)]=\left[\begin{array}{cc}
\mathbf{I}_{n}+\frac{\left(a_{\ell}-1\right)}{\left\|\mathbf{f}_{\ell}\right\|^{2}} \mathbf{f}_{\ell} \mathbf{f}_{\ell}^{\mathrm{T}} & \frac{b_{\ell} \mathbf{f}_{\ell}}{\left\|\mathbf{f}_{\ell}\right\|} \\
\frac{b_{\ell} \mathbf{f}_{\ell}^{\mathrm{T}}}{\left\|\mathbf{f}_{\ell}\right\|} & a_{\ell}
\end{array}\right],
$$

where

$$
\begin{aligned}
& a_{\ell}:=\cosh \left(\frac{\Delta t\left\|\mathbf{f}_{\ell}\right\|}{\left\|\mathbf{u}_{\ell}\right\|}\right), \\
& b_{\ell}:=\sinh \left(\frac{\Delta t\left\|\mathbf{f}_{\ell}\right\|}{\left\|\mathbf{u}_{\ell}\right\|}\right) .
\end{aligned}
$$

Substituting (3.13) for $\mathrm{G}(\ell)$ into (3.9), we obtain

$$
\begin{gathered}
\mathbf{u}_{\ell+1}=\mathbf{u}_{\ell}+\eta_{\ell} \mathbf{f}_{\ell} \\
\left\|\mathbf{u}_{\ell+1}\right\|=a_{\ell}\left\|\mathbf{u}_{\ell}\right\|+\frac{b_{\ell}}{\left\|\mathbf{f}_{\ell}\right\|} \mathbf{f}_{\ell} \cdot \mathbf{u}_{\ell}
\end{gathered}
$$

where

$$
\eta_{\ell}:=\frac{b_{\ell}\left\|\mathbf{u}_{\ell}\right\|\left\|\mathbf{f}_{\ell}\right\|+\left(a_{\ell}-1\right) \mathbf{f}_{\ell} \cdot \mathbf{u}_{\ell}}{\left\|\mathbf{f}_{\ell}\right\|^{2}}
$$

is an adaptive factor. From $\mathbf{f}_{\ell} \cdot \mathbf{u}_{\ell} \geq-\left\|\mathbf{f}_{\ell}\right\|\left\|\mathbf{u}_{\ell}\right\|$, we can prove that

$$
\eta_{\ell} \geq\left[1-\exp \left(-\frac{\Delta t\left\|\mathbf{f}_{\ell}\right\|}{\left\|\mathbf{u}_{\ell}\right\|}\right)\right] \frac{\left\|\mathbf{u}_{\ell}\right\|}{\left\|\mathbf{f}_{\ell}\right\|}>0 \quad \forall \Delta t>0 .
$$

This scheme is group properties preserved for all $\Delta t>0$. 


\subsection{One-step GPS}

Applying scheme (3.15) on (2.8), we can compute the heat conduction equation by the GPS. Assume that the total time $T$ is divided by $K$ steps, that is, the time step size we use in the GPS is $\Delta t=T / K$.

Starting from an initial augmented condition $\mathbf{X}_{0}=\mathbf{X}(0)$, we may want to calculate the value $\mathbf{X}(T)$ at a desired time $t=T$. By (3.9), we can obtain that

$$
\mathbf{X}_{T}=\mathrm{G}_{K}(\Delta t) \cdots \mathrm{G}_{1}(\Delta t) \mathbf{X}_{0}
$$

where $\mathbf{X}_{T}$ approximates the real $\mathbf{X}(T)$ within a certain accuracy depending on $\Delta t$. However, let us recall that each $\mathrm{G}_{i}, i=1, \ldots, K$, is an element of the Lie-group $S O_{o}(n, 1)$, and by the closure property of Lie-group, $\mathrm{G}_{K}(\Delta t) \cdots \mathrm{G}_{1}(\Delta t)$ is also a Lie-group denoted by $\mathrm{G}(T)$. Hence, we have

$$
\mathbf{X}_{T}=\mathbf{G}(T) \mathbf{X}_{0}
$$

This is a one-step transformation from $\mathbf{X}_{0}$ to $\mathbf{X}_{T}$.

Usually, it is very hard to find an exact solution of $\mathrm{G}(T)$; however, a numerical one may be obtained approximately without any difficulty. The most simple method to calculate $\mathrm{G}(T)$ is given by

$$
\mathbf{G}(T)=\left[\begin{array}{cc}
\mathbf{I}_{n}+\frac{(a-1)}{\left\|\mathbf{f}_{0}\right\|^{2}} \mathbf{f}_{0} \mathbf{f}_{0}^{\mathrm{T}} & \frac{b \mathbf{f}_{0}}{\left\|\mathbf{f}_{0}\right\|} \\
\frac{b \mathbf{f}_{0}^{\mathrm{T}}}{\left\|\mathbf{f}_{0}\right\|} & a
\end{array}\right],
$$

where

$$
\begin{aligned}
& a:=\cosh \left(\frac{T\left\|\mathbf{f}_{0}\right\|}{\left\|\mathbf{u}_{0}\right\|}\right), \\
& b:=\sinh \left(\frac{T\left\|\mathbf{f}_{0}\right\|}{\left\|\mathbf{u}_{0}\right\|}\right) .
\end{aligned}
$$

Then from (3.15) and (3.16), we obtain a one-step GPS:

$$
\begin{gathered}
\mathbf{u}_{T}=\mathbf{u}_{0}+\eta \mathbf{f}_{0} \\
\left\|\mathbf{u}_{T}\right\|=a\left\|\mathbf{u}_{0}\right\|+\frac{b \mathbf{f}_{0} \cdot \mathbf{u}_{0}}{\left\|\mathbf{f}_{0}\right\|}
\end{gathered}
$$

where

$$
\eta=\frac{(a-1) \mathbf{f}_{0} \cdot \mathbf{u}_{0}+b\left\|\mathbf{u}_{0}\right\|\left\|\mathbf{f}_{0}\right\|}{\left\|\mathbf{f}_{0}\right\|^{2}}
$$




\section{Identifying $q(x)$ by the LGEM}

In this section, we will start to estimate the potential function $q(x)$. By using the one-step GPS, we also suppose that the initial value of $u(x, 0)=y(x)$ is given and its corresponding eigenvalue is known.

Applying the one-step GPS in (3.23) on (2.8) from time $t=0$ to time $t=T$, we obtain a nonlinear equation for $q_{i}$ :

$$
u_{i}^{T}=u_{i}^{0}+\frac{\eta p_{i}}{(\Delta x)^{2}}\left(u_{i+1}^{0}-2 u_{i}^{0}+u_{i-1}^{0}\right)+\eta p_{i}^{\prime} \frac{u_{i+1}^{0}-u_{i}^{0}}{\Delta x}+\eta\left(q_{i}+\lambda r_{i}\right) u_{i}^{0}+\eta h_{i}(0) .
$$

It is not difficult to rewrite (4.1) as

$$
q_{i}=\frac{1}{u_{i}^{0}}\left[\frac{u_{i}^{T}-u_{i}^{0}}{\eta}-\frac{p_{i}}{(\Delta x)^{2}}\left(u_{i+1}^{0}-2 u_{i}^{0}+u_{i-1}^{0}\right)-\frac{p_{i}^{\prime}}{\Delta x}\left(u_{i+1}^{0}-u_{i}^{0}\right)-\lambda r_{i} u_{i}^{0}-h_{i}(0)\right],
$$

noting that $\eta$ in the above is not a constant but a nonlinear function of $q_{i}$ as shown by (3.25). Therefore, in this stage, we cannot calculate $q_{i}$ by a simple equation. However, we will prove below that $\eta$ is fully determined by $u_{i}^{0}$ and $u_{i}^{T}$.

In order to solve $q_{i}$, let us return to (3.23):

$$
\mathbf{f}_{0}=\frac{1}{\eta}\left[\mathbf{u}_{T}-\mathbf{u}_{0}\right] .
$$

Substituting it for $\mathbf{f}_{0}$ into (3.24), we obtain

$$
\frac{\left\|\mathbf{u}_{T}\right\|}{\left\|\mathbf{u}_{0}\right\|}=a+\frac{b\left[\mathbf{u}_{T}-\mathbf{u}_{0}\right] \cdot \mathbf{u}_{0}}{\left\|\mathbf{u}_{T}-\mathbf{u}_{0}\right\|\left\|\mathbf{u}_{0}\right\|}
$$

where

$$
\begin{aligned}
& a:=\cosh \left(\frac{T\left\|\mathbf{u}_{T}-\mathbf{u}_{0}\right\|}{\eta\left\|\mathbf{u}_{0}\right\|}\right), \\
& b:=\sinh \left(\frac{T\left\|\mathbf{u}_{T}-\mathbf{u}_{0}\right\|}{\eta\left\|\mathbf{u}_{0}\right\|}\right) .
\end{aligned}
$$

Let

$$
\begin{aligned}
\cos \theta & :=\frac{\left[\mathbf{u}_{T}-\mathbf{u}_{0}\right] \cdot \mathbf{u}_{0}}{\left\|\mathbf{u}_{T}-\mathbf{u}_{0}\right\|\left\|\mathbf{u}_{0}\right\|} \\
S & :=\frac{T\left\|\mathbf{u}_{T}-\mathbf{u}_{0}\right\|}{\left\|\mathbf{u}_{0}\right\|}
\end{aligned}
$$

and from (4.4)-(4.6), it follows that

$$
\frac{\left\|\mathbf{u}_{T}\right\|}{\left\|\mathbf{u}_{0}\right\|}=\cosh \left(\frac{S}{\eta}\right)+\cos \theta \sinh \left(\frac{S}{\eta}\right)
$$


Upon defining

$$
Z:=\exp \left(\frac{S}{\eta}\right)
$$

and from (4.9), we obtain a quadratic equation for $Z$ :

$$
(1+\cos \theta) Z^{2}-\frac{2\left\|\mathbf{u}_{T}\right\|}{\left\|\mathbf{u}_{0}\right\|} Z+1-\cos \theta=0 .
$$

The solution is found to be

$$
Z=\frac{\left\|\mathbf{u}_{T}\right\| /\left\|\mathbf{u}_{0}\right\| \pm \sqrt{\left(\left\|\mathbf{u}_{T}\right\| /\left\|\mathbf{u}_{0}\right\|\right)^{2}-\left(1-\cos ^{2} \theta\right)}}{1+\cos \theta} \quad \text { if } \pm \cos \theta>0 ;
$$

and from (4.10), we obtain a closed-form solution of $\eta$ :

$$
\eta=\frac{T\left\|\mathbf{u}_{T}-\mathbf{u}_{0}\right\|}{\left\|\mathbf{u}_{0}\right\| \ln Z}
$$

Up to here, we must point out that for a given $T, \eta$ is fully determined by $\mathbf{u}_{0}$ and $\mathbf{u}_{T}$ which are supposed to be known. Therefore, the original nonlinear equation (4.2) becomes a linear equation for $q_{i}$.

By using (2.1), we have

$$
u_{i}^{T}=(1+T) u_{i}^{0}=(1+T) y_{i}
$$

and thus the vector $\mathbf{u}_{T}$ is proportional to $\mathbf{u}_{0}$ with a multiplier $1+T$ larger than 1 . Under this condition, we have $\cos \theta=1$, and $Z$ is given by

$$
Z=\frac{\left\|\mathbf{u}_{T}\right\|}{\left\|\mathbf{u}_{0}\right\|}=1+T
$$

and hence from (4.13), we have

$$
\eta=\frac{T^{2}}{\ln (1+T)}
$$

Inserting (4.14) and (4.16) into (4.2), we obtain a very simple formula to estimate $q_{i}$ by

$$
q_{i}=\frac{1}{y_{i}}\left[\frac{y_{i} \ln (1+T)}{T}-\frac{p_{i}}{(\Delta x)^{2}}\left(y_{i+1}-2 y_{i}+y_{i-1}\right)-\frac{p_{i}^{\prime}}{\Delta x}\left(y_{i+1}-y_{i}\right)-\lambda r_{i} y_{i}-y_{i}+F_{i}\right] \text {. }
$$

This solution is in a closed-form for $q_{i}$.

In the above, we have mentioned that $\eta$ is a nonlinear function of $q_{i}$; however, by viewing (4.7), (4.12), and (4.13), it is known that $\eta$ is fully determined by $\mathbf{u}_{0}$ and $\mathbf{u}_{T}$. Furthermore, by using (4.14) $\eta$ becomes a constant given by (4.16). This point is very important for our closedform solution of parameter. The key points rely on the construction of the method by using the one-step GPS for the estimation of parameter, and the full use of the $n+1$ equations (3.23) and (3.24). To distinguish the present method by a joint use of the one-step GPS and the closed-form solution with the aid of (3.24), we may call the new method a Lie-group estimation method (LGEM). 


\section{Applying the LGEM to estimate $p(x)$}

In this section, we will derive a simple linear equations system to solve the coefficients $p_{i}, i=$ $1, \ldots, n$. However, for simplicity, we assume that $q(x)=r(x)=0$ in this section.

A similar finite difference as that used in (2.6) for $u^{\prime}(x)$ can be used for $p^{\prime}(x)$ in (2.8). In doing so, we can obtain a system of ODEs for $u$ with $t$ as an independent variable:

$$
\dot{u}_{i}(t)=\frac{p_{i+1}-p_{i}}{\Delta x} \frac{u_{i+1}(t)-u_{i}(t)}{\Delta x}+p_{i} \frac{u_{i+1}(t)-2 u_{i}(t)+u_{i-1}(t)}{(\Delta x)^{2}}+h_{i}(t)
$$

The known initial condition is given by

$$
u_{i}^{0}=y\left(x_{i}\right), \quad i=1, \ldots, n,
$$

which is obtained from (2.1) by a discretization.

Applying the same idea of LGEM on (5.1), we can obtain a closed-form formula to estimate $p_{i}$ :

$$
p_{i}=\frac{(\Delta x)^{2}}{u_{i}^{0}-u_{i-1}^{0}}\left[\frac{u_{i+1}^{0}-u_{i}^{0}}{(\Delta x)^{2}} p_{i+1}+h_{i}(0)-\frac{1}{\eta}\left(u_{i}^{T}-u_{i}^{0}\right)\right]
$$

and, moreover, by using the data of $u_{i}^{T}$ given by (4.14) and (4.16) for $\eta$, we can derive a much simple equation for $p_{i}$ :

$$
p_{i}=\frac{(\Delta x)^{2}}{y_{i}-y_{i-1}}\left[\frac{y_{i+1}-y_{i}}{(\Delta x)^{2}} p_{i+1}+y_{i}-F_{i}-\frac{y_{i} \ln (1+T)}{T}\right]
$$

This will be called a closed-form estimation method. The above equation can be used sequentially to find $p_{i}, i=n, \ldots, 1$, if we know $p_{n+1}$ a priori. Here, $p_{n+1}$ is the right-end boundary value of $p(x)$, and is supposed to be known for simplicity.

However, we can develop another estimation method through iterations. The numerical procedures for estimating $p_{i}$ are described as follows. We assume an initial value of $p_{i}$, for example, $p_{i}=1$. Substituting it into (5.1), we can apply the GPS to integrate it from $t=0$ to $t=T$ through $T / \Delta t$ steps. Then, we obtain $u_{i}^{T}$. Inserting it into (5.3), we can calculate a new $p_{i}$, which is then compared with the old $p_{i}$. If the difference of these two sets of $p_{i}$ is smaller than a given criterion, then we stop the iteration and the final $p_{i}$ is obtained.

The processes are summarized as follows:

(i) give an initial $p_{i}=1$;

(ii) for $j=1,2 \ldots$, we repeat the following calculations; calculate $u_{i}^{T}$ by using the GPS in (3.15) to integrate (5.1) from $t=0$ to $t=T$, where $\mathbf{f}$ is a vector form of the right-hand side of (5.1); 
(iii) insert the above calculated $u_{i}^{T}$ denoted by $u_{i}^{T}(j)$ together with $u_{i}^{0}$ given by (5.2) into

$$
p_{i}^{j}=\frac{(\Delta x)^{2}}{u_{i}^{0}-u_{i-1}^{0}}\left[\frac{u_{i+1}^{0}-u_{i}^{0}}{(\Delta x)^{2}} p_{i+1}^{j}-\frac{1}{\eta^{j}}\left[u_{i}^{T}(j)-u_{i}^{0}\right]+h_{i}(0)\right],
$$

where $\eta^{j}$ is calculated from (4.13) by substituting $\mathbf{u}_{T}^{j}$ for each step. If $p_{i}^{j}$ converges according to a given stopping criterion:

$$
\sum_{i=1}^{n}\left(p_{i}^{j+1}-p_{i}^{j}\right)^{2}<\varepsilon^{2},
$$

then stop, otherwise, go to step (ii).

Basically, the present method has repeatedly used the time direction integration of (5.1) to obtain the final time data to adjust $p_{i}$, which will be called an iterative estimation method.

\section{Numerical examples}

Example 6.1. For a first example, we consider an inverse Sturm-Liouville problem to identify the potential function in the Schrödinger equation:

$$
\begin{gathered}
y^{\prime \prime}(x)+\left(\beta-\alpha^{2} x^{2}\right) y(x)=0, \\
y(-\infty)=y(\infty)=0 .
\end{gathered}
$$

Here, $\beta=(2 k+1) \alpha$ is the eigenvalue, and

$$
y_{k}(x)=H_{k}(x) \exp \left(-\frac{\alpha x^{2}}{2}\right)
$$

is the eigenfunction, where the Hermite polynomials for $k=0,1,2,3,4$ are given by

$$
\begin{aligned}
& H_{0}(x)=1 \\
& H_{1}(x)=2 x \\
& H_{2}(x)=-2+4 x^{2} \\
& H_{3}(x)=-12 x+8 x^{3} \\
& H_{4}(x)=12-48 x^{2}+16 x^{4} .
\end{aligned}
$$

In general, $H_{k}(x)=(-1)^{k} e^{x^{2}} d^{k} e^{-x^{2}} / d x^{k}$.

In order to recover the potential function $q(x)$ from the given eigenvalue and eigenfunction, we apply (4.17) on this problem by taking $p=1, p^{\prime}=0, r=1$, and $F=0$. We also take $x_{0}=-5$ and $x_{f}=5$ and let $\Delta x=10 / 300, \alpha=1$, and $T=0.0001$.

The estimation errors of $q(x)$ are shown in Figures $1(\mathrm{a})$ and $1(\mathrm{~b})$, for $k=1$ and $y_{1}(x)$ and $k=3$ and $y_{3}(x)$ as the inputs on (4.17). From these two figures, it can be seen that the estimations of $q(x)$ are quite accurate. However, near the boundaries, the errors are increased. In order to avoid the boundary effect on the estimation of $q(x)$, we can extend the range of $x$ into a larger one. 


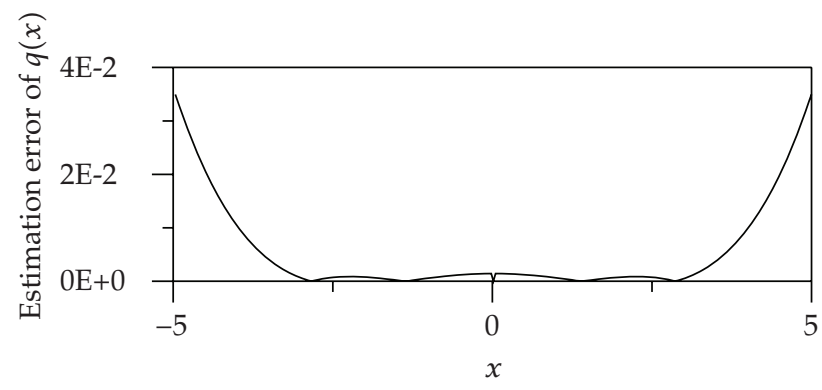

(a)

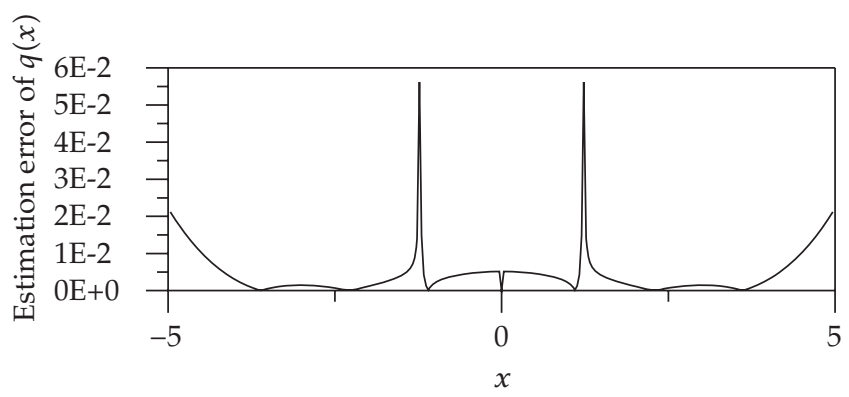

(b)

Figure 1: Example 6.1 of identifying the Schrödinger equation (a) plotting the estimation error by using the second spectral function, while (b) by using the fourth spectral function.

Example 6.2. For a second test example, we consider the Sturm-Liouville problem with

$$
\begin{gathered}
{\left[x^{-1} y^{\prime}(x)\right]^{\prime}+(\lambda+1) x^{-3} y(x)=0,} \\
y(1)=y(e)=0 .
\end{gathered}
$$

The eigenvalue is $\lambda_{k}=k^{2} \pi^{2}, k \in \mathbb{N}$, and the eigenfunction is $y_{k}=a x \sin (k \pi \ln x)$, where $a$ is an arbitrary nonzero constant fixed to be $a=100$.

In Figure 2, we compare the estimation errors by considering the noisy data with $y_{\sigma}=$ ax $\sin (\pi \ln x)+\sigma R(i)$, where $R(i)$ are random numbers between -1 and +1 . The $L^{2}$ error for $\sigma=0$ is about 0.327 while that for $\sigma=0.01$ is about 0.373 .

Example 6.3. In this example, we estimate $p(x)$ by setting $q(x)=0$ and $r(x)=0$. Let us use the following example to demonstrate the process in Section 5. This example is given by

$$
\begin{gathered}
p(x)=(x-3)^{2}, \\
F(x)=6(x-3)^{2}, \quad h(x, t)=y(x)-(1+t) F(x)=-(x-3)^{2}(5+6 t) .
\end{gathered}
$$

Under the boundary conditions

$$
u(0, t)=9(1+t), \quad u(1, t)=4(1+t)
$$

and the initial condition

$$
u(x, 0)=(x-3)^{2},
$$




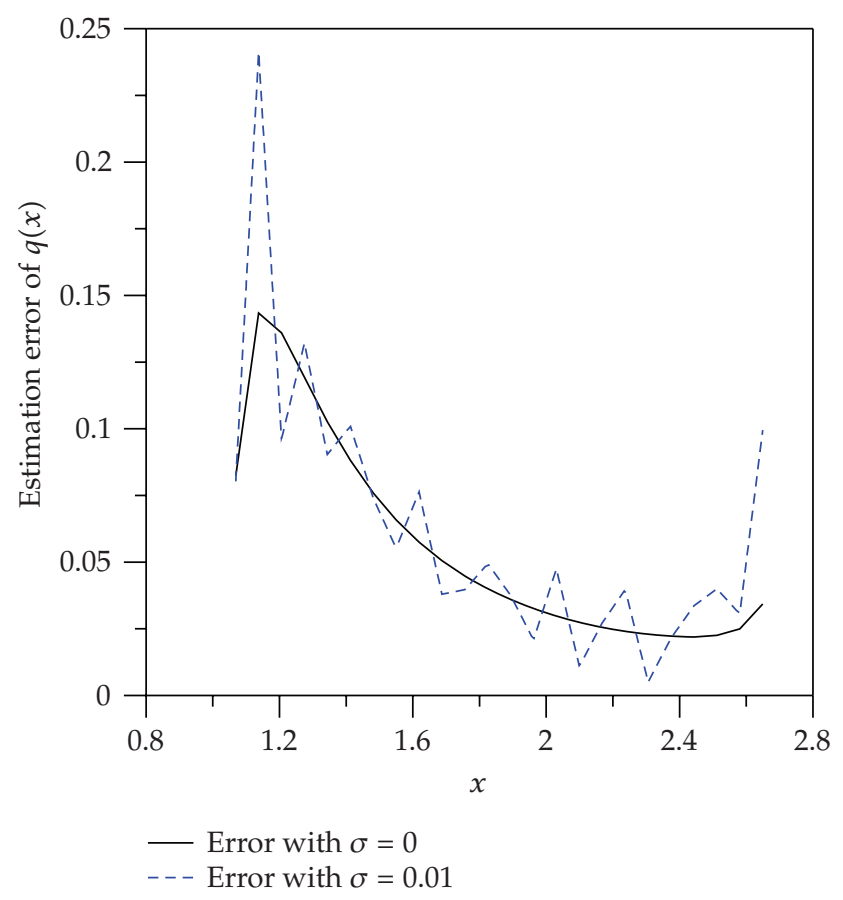

Figure 2: Example 6.2 of identifying $q(x)$, the estimation errors were plotted.

the exact solution is given by

$$
u(x, t)=(x-3)^{2}(1+t)
$$

We apply the LGEM on this identification of $p(x)$, where we have fixed $\Delta x=1 / 30$, $\Delta t=5 \times 10^{-5}$, and $T=0.01$. Under the stopping criterion with $\epsilon=10^{-3}$, the process is convergent within 34 iterations. In Figure 3(a), we plot the tentative $p_{i}$ for the first iteration, the fifth iteration, the tenth iteration, and the fifteenth iteration, the last of which is already close to the exact solution. The numerical solutions of $p_{i}$ are close to the exact ones with the $L^{2}$ error about 0.0156, and the maximum relative error about $4 \times 10^{-3}$ as shown in Figure 3(b).

Example 6.4. The following example has been calculated by Keung and Zou [12] using the augmented Lagrange method:

$$
\begin{gathered}
p(x)=3+2 x^{2}-2 \sin (2 \pi x), \\
F(x)=2 \pi[4 x-4 \pi \cos (2 \pi x)] \cos (2 \pi x)-4 \pi^{2}\left[3+2 x^{2}-2 \sin (2 \pi x)\right] \sin (2 \pi x) .
\end{gathered}
$$

The exact solution is given by

$$
u(x, t)=(1+t) \sin (2 \pi x) .
$$

We first apply the iterative LGEM on this identification of $p(x)$, where we have fixed $\Delta x=2 / 60, \Delta t=10^{-5}$, and $T=0.003$. Under the stopping criterion with $\epsilon=10^{-3}$, the process is convergent within 37 iterations. In Figure 4, we compare the estimated solution with exact solution. For this estimation, we have a maximum error with 0.377 and an $L^{2}$ error with 1.62. 


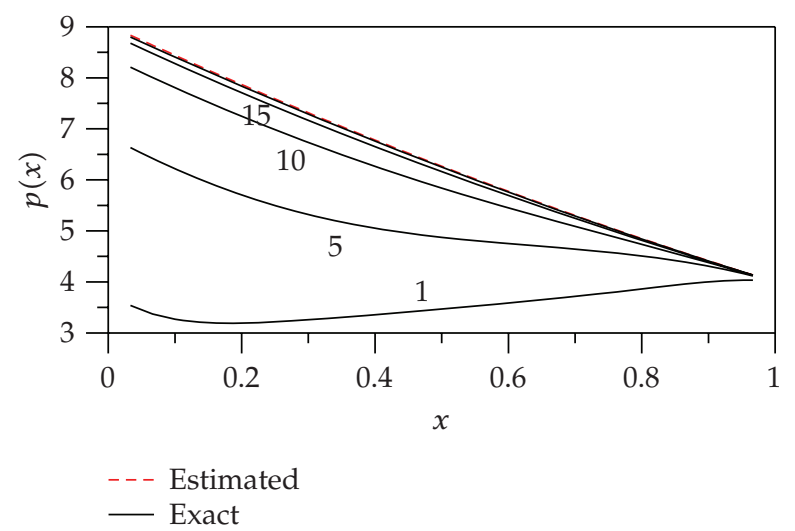

(a)

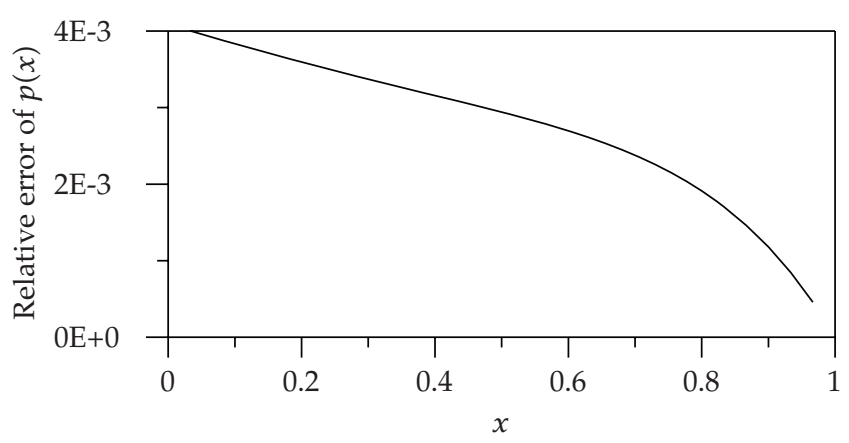

(b)

Figure 3: Example 6.3 by using an iterative method: (a) comparing estimated and exact $p(x)$, and (b) plotting the relative error of estimation.

On the other hand, we also apply (5.4) on this estimation by using $\Delta x=2.2 / 150$ and $T=0.001$, of which the result is shown in Figure 4 by the dashed-dotted line. For the later estimation, we have a maximum error with 0.59 and an $L^{2}$ error with 1.402 . It is slightly more accurate than the iterative method; however, there appear four kinks near the extremal points of $p(x)$, where $p^{\prime}(x)=0$.

Example 6.5. For this example, we consider a simple exact $y(x)=\sin (\pi x)$ under a noise given by $y_{\delta}=\sin (\pi x)+\delta \cos (3 \pi x)$. The following results are used:

$$
\begin{aligned}
& p(x)=x, \\
& F(x)=\pi \cos (\pi x)-\pi^{2} x \sin (\pi x) .
\end{aligned}
$$

For the integration in (1.5), we have employed the trapezoidal rule to calculate $y_{\delta}^{\prime}(x)$ at discretized spatial points. Then inserting them into (1.4), we can obtain $p(x)$ by the ND method under $\delta=0$ and $\delta=0.02$, whose errors are shown in Figure 5(a). The best parameter $\alpha$ is fixed to be 0.95 for $\delta=0$ and 0.85 for $\delta=0.02$, not 0.0085 as that used by Ahn et al. [15].

On the other hand, we also apply (5.4) on this estimation of $p(x)$ by using $\Delta x=0.01$ and $T=0.01$, of which the errors under $\delta=0$ and $\delta=0.02$ are shown in Figure 5(a). It can be seen that the maximum errors of LGEM are much smaller than that obtained by the ND method. Especially, when $\delta=0$ the LGEM with all its absolute error is smaller than that by the ND method. 


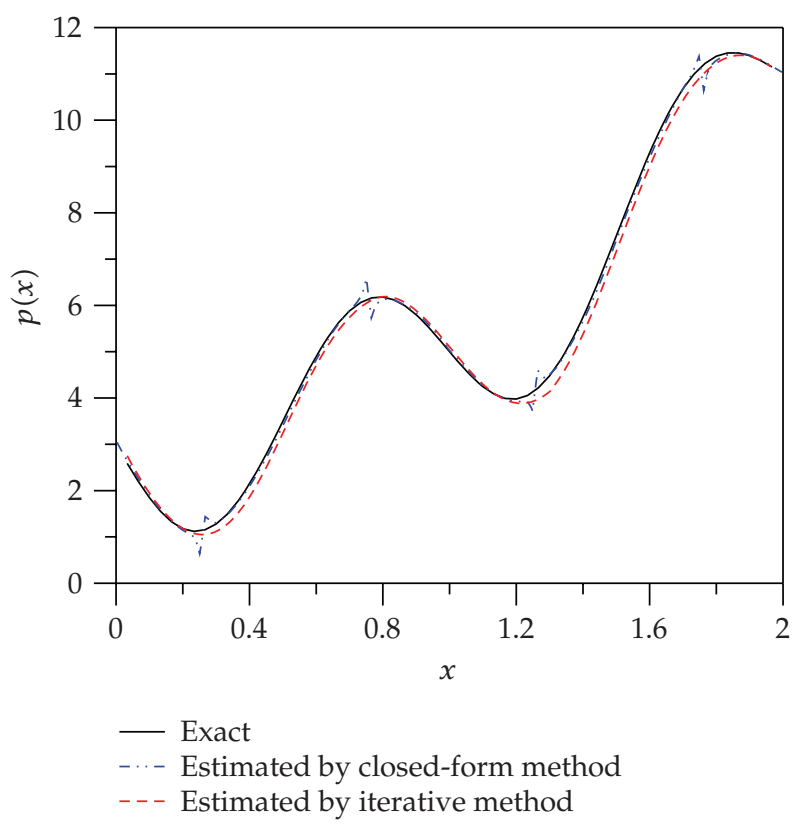

Figure 4: In Example 6.4 we compare exact $p(x)$ with the estimated ones by using an iterative method and a closed-form method.

For both methods applied in this example, we also consider the random noise disturbance given by $y_{\sigma}=\sin \left(\pi x_{i}\right)+\sigma R(i)$, where $R(i)$ are random numbers between -1 and +1 . In Figure 5(b), we compare the numerical errors by applying the LGEM and ND for this case under a noise with $\sigma=0.001$. For the ND, the best parameter of $\alpha$ is $\alpha=0.5$. Obviously, the absolute error of LGEM is smaller than that given by the ND method. This also shows that the method of LGEM can be against the random and also definite disturbances.

Example 6.6. Let us consider the following linear BVP:

$$
x y^{\prime \prime}(x)+y^{\prime}(x)=F(x), \quad y(1)=0, y(2)=2,
$$

where $F(x)=x^{3 / 2} \cos x$ has no closed-form integral of $\int_{1}^{x} F(s) d s$, such that $y(x)$ has also no closed-form solution. In this case, we applied the Lie-group shooting method [20] to calculate $y$.

In (1.4), we require to know both $p\left(x_{0}\right)$ and $y^{\prime}\left(x_{0}\right)$. This is a great drawback of the ND method. Because the calculated data of $y$ are discretized, we may approximate $y^{\prime}\left(x_{0}\right)$ by $\left(y_{1}-y_{0}\right) / \Delta x$. Then, inserting the calculated data of $y$ into (1.4), we can obtain $p(x)$ by the ND method under $\sigma=0$ and $\sigma=0.001$, whose errors, as shown in Figure 6, are very large. The best parameter $\alpha$ is fixed to be 0.45 for $\sigma=0$ and 0.43 for $\sigma=0.001$.

Then, we apply (5.4) on this estimation of $p(x)$ by using $\Delta x=0.01$ and $T=0.01$ of which the errors under $\sigma=0$ and $\sigma=0.02$ are shown in Figure 6. The errors of LGEM are much smaller than that obtained by the ND method with a ratio $10^{-4}$ of these two maximum errors for the un-noised case and $6.7 \times 10^{-3}$ for the noised case. 


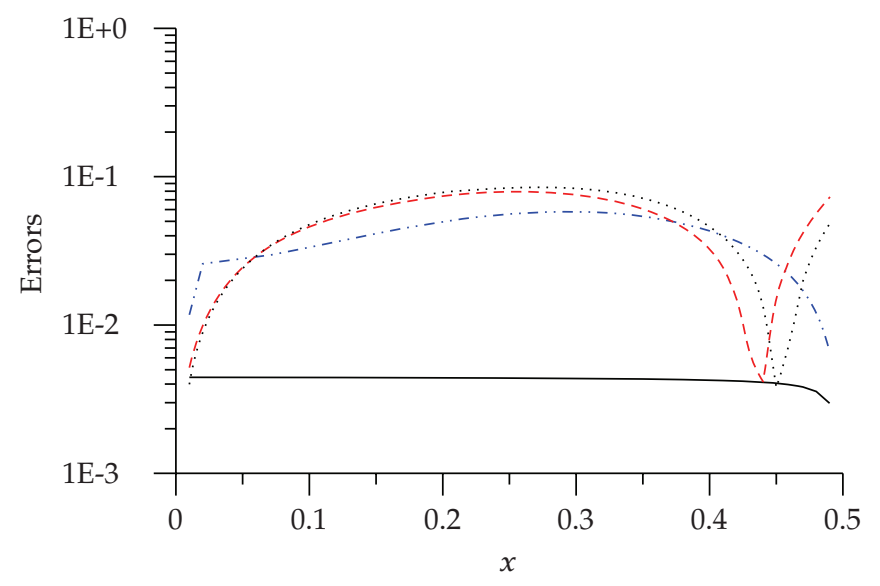

E.... Error of ND with $\delta=0.02$

- - - Error of ND with $\delta=0$

-.- Error of LGEM with $\delta=0.02$

- Error of LGEM with $\delta=0$

(a)

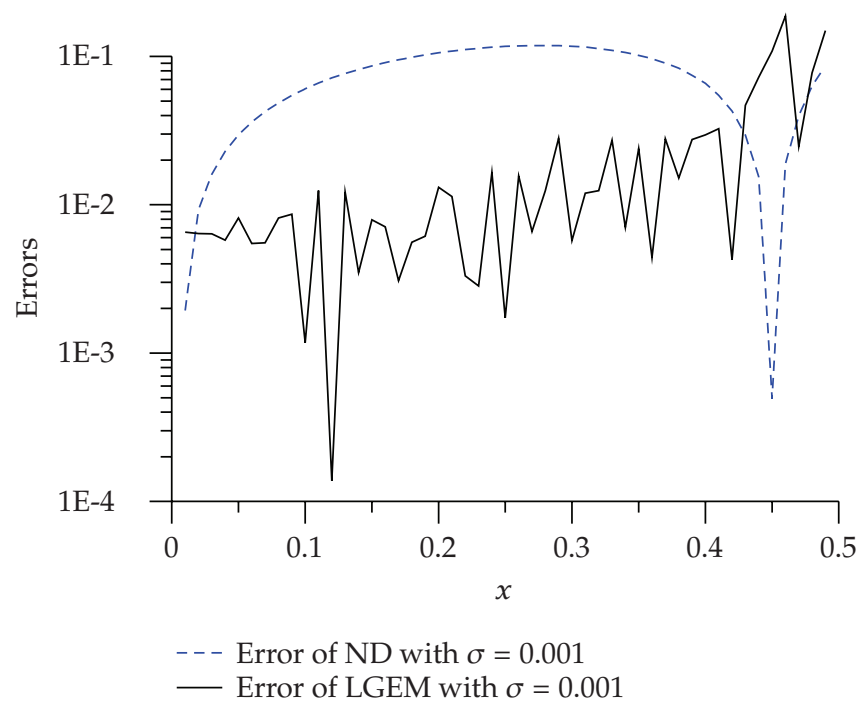

(b)

Figure 5: Example 6.5 comparing the estimation errors of $p(x)$ : (a) definite disturbances with $\delta=0,0.02$, and (b) random disturbance with $\sigma=0.001$.

\section{Conclusions}

In order to estimate the potential function under a given spectral function and its corresponding eigenvalue, we have employed the LGEM to derive an algebraic equation and solved it in a closed form. We transformed the inverse Sturm-Liouville problem into a parameter identification problem for a parabolic type PDE, and then established a one-step GPS for the semidiscretization of that PDE. We also established an iterative method to estimate the unknown coefficient in a second-order Sturm-Liouville operator. 


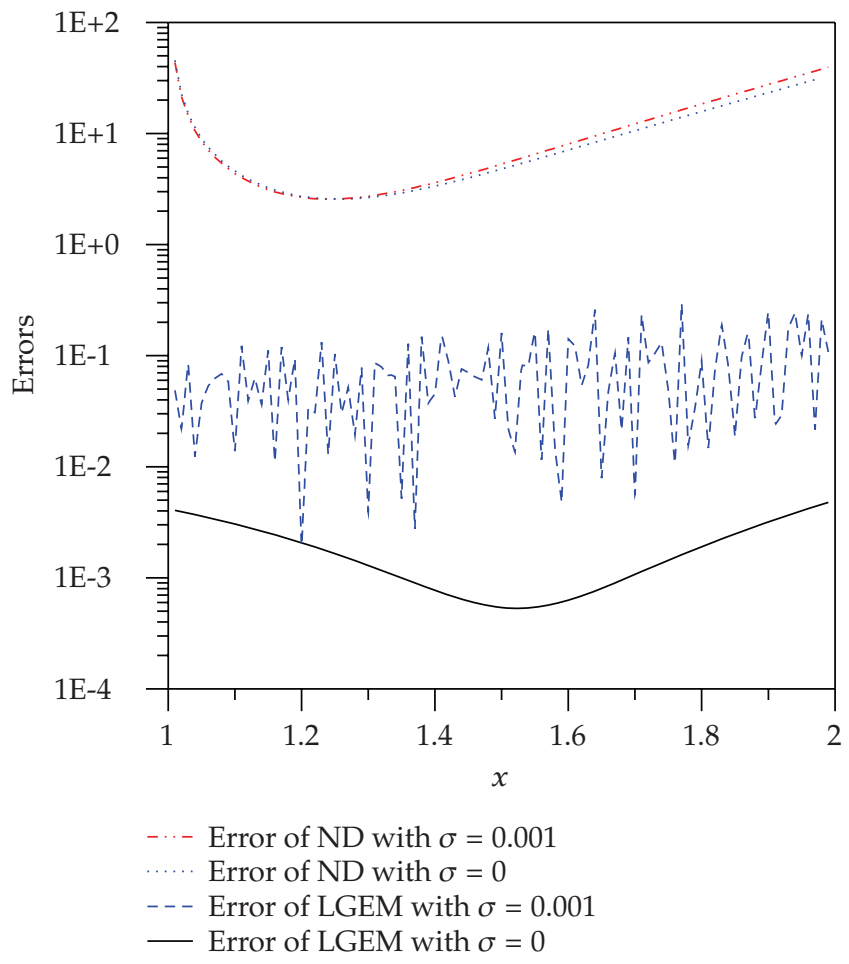

Figure 6: Example 6.6 comparing the estimation errors of $p(x)$ under random disturbances with $\sigma=0$ and $\sigma=0.001$.

Numerical examples were worked out, which show that the new LGEM is applicable for the estimations of unknown functions. When disturbances are exerted on the input data, we also verified that the present approach can be against them very well. The tested case shows that the LGEM is superior than the ND method examined here. Through this study, it can be concluded that the new estimation method is accurate, effective, and stable. Its numerical implementation is very simple and the computational speed is very fast.

\section{References}

[1] I. M. Gel'fand and B. M. Levitan, "On the determination of a differential equation from its spectral function," American Mathematical Society Translations, vol. 1, pp. 253-304, 1955.

[2] J. R. McLaughlin, "Analytical methods for recovering coefficients in differential equations from spectral data," SIAM Review, vol. 28, no. 1, pp. 53-72, 1986.

[3] A. L. Andrew, "Numerical solution of inverse Sturm-Liouville problems," The Australian \& New Zealand Industrial and Applied Mathematics Journal, vol. 45, pp. C326-C337, 2004.

[4] J. Paine, "A numerical method for the inverse Sturm-Liouville problem," SIAM Journal on Scientific and Statistical Computing, vol. 5, no. 1, pp. 149-156, 1984.

[5] M. T. Chu, "Inverse eigenvalue problems," SIAM Review, vol. 40, no. 1, pp. 1-39, 1998.

[6] J. R. McLaughlin, "Inverse spectral theory using nodal points as data-a uniqueness result," Journal of Differential Equations, vol. 73, no. 2, pp. 354-362, 1988.

[7] P. J. Browne and B. D. Sleeman, "Inverse nodal problems for Sturm-Liouville equations with eigenparameter dependent boundary conditions," Inverse Problems, vol. 12, no. 4, pp. 377-381, 1996.

[8] Y.-H. Cheng, C. K. Law, and J. Tsay, "Remarks on a new inverse nodal problem," Journal of Mathematical Analysis and Applications, vol. 248, no. 1, pp. 145-155, 2000. 
[9] O. H. Hald and J. R. McLaughlin, "Solutions of inverse nodal problems," Inverse Problems, vol. 5, no. 3, pp. 307-347, 1989.

[10] X.-F. Yang, "A solution of the inverse nodal problem," Inverse Problems, vol. 13, no. 1, pp. 203-213, 1997.

[11] D. Boley and G. H. Golub, "A survey of matrix inverse eigenvalue problems," Inverse Problems, vol. 3, no. 4, pp. 595-622, 1987.

[12] Y. L. Keung and J. Zou, "An efficient linear solver for nonlinear parameter identification problems," SIAM Journal on Scientific Computing, vol. 22, no. 5, pp. 1511-1526, 2000.

[13] J. Li, "General explicit difference formulas for numerical differentiation," Journal of Computational and Applied Mathematics, vol. 183, no. 1, pp. 29-52, 2005.

[14] C. Shu, Differential Quadrature and Its Application in Engineering, Springer, London, UK, 2000.

[15] S. Ahn, U. J. Choi, and A. G. Ramm, "A scheme for stable numerical differentiation," Journal of Computational and Applied Mathematics, vol. 186, no. 2, pp. 325-334, 2006.

[16] A. Iserles and A. Zanna, "Preserving algebraic invariants with Runge-Kutta methods," Journal of Computational and Applied Mathematics, vol. 125, no. 1-2, pp. 69-81, 2000.

[17] C.-S. Liu, "New integrating methods for time-varying linear systems and Lie-group computations," Computer Modeling in Engineering \& Sciences, vol. 20, no. 3, pp. 157-175, 2007.

[18] A. Iserles, H. Z. Munthe-Kaas, S. P. Nørsett, and A. Zanna, "Lie-group methods," Acta Numerica, vol. 9, pp. 215-365, 2000.

[19] C.-S. Liu, "Cone of non-linear dynamical system and group preserving schemes," International Journal of Non-Linear Mechanics, vol. 36, no. 7, pp. 1047-1068, 2001.

[20] C.-S. Liu, "The Lie-group shooting method for nonlinear two-point boundary value problems exhibiting multiple solutions," Computer Modeling in Engineering \& Sciences, vol. 13, no. 2, pp. 149-163, 2006.

[21] C.-S. Liu, "One-step GPS for the estimation of temperature-dependent thermal conductivity," International Journal of Heat and Mass Transfer, vol. 49, no. 17-18, pp. 3084-3093, 2006.

[22] C.-S. Liu, "An efficient simultaneous estimation of temperature-dependent thermophysical properties," Computer Modeling in Engineering \& Sciences, vol. 14, no. 2, pp. 77-90, 2006.

[23] C.-S. Liu, "Identification of temperature-dependent thermophysical properties in a partial differential equation subject to extra final measurement data," Numerical Methods for Partial Differential Equations, vol. 23, no. 5, pp. 1083-1109, 2007.

[24] C.-S. Liu, L.-W. Liu, and H.-K. Hong, "Highly accurate computation of spatial-dependent heat conductivity and heat capacity in inverse thermal problem," Computer Modeling in Engineering $\mathcal{E}$ Sciences, vol. 17, no. 1, pp. 1-18, 2007. 